\title{
THE EFFECTS OF DIFFERENT ROOTSTOCKS ON AROMA VOLATILE CONSTITUENTS IN THE FRUITS OF 'FUJI' APPLES (MALUS DOMESTICA BORKH.)
}

\author{
GUR, E. \\ Canakkale Onsekiz, Mart University, Faculty of Agriculture, Department of Horticulture \\ 17020 Canakkale, Turkey \\ (e-mail: engingur@comu.edu.tr; phone:+90-(286)-218-00-18) \\ (Received 22 $2^{\text {nd }}$ Apr 2019; accepted $4^{\text {th }}$ Jul 2019)
}

\begin{abstract}
Five-year-old 'Fuji' (Malus domestica Borkh.) apple trees grafted on MM-106, M-9, M-26, and MM-111 in a commercial orchard in Çanakkale (Turkey), were used for determination of fruit quality and aroma volatiles. Fruits were sampled at the commercial harvesting stage at the end of September in 2015 and 2016. Fruit quality characteristics and aroma volatiles changed significantly in apples grown on four different rootstocks. The largest fruits (212.2 g) with highest total soluble solids (18.40\%) were obtained from the trees on M9 rootstock. According to chromatography analysis, a total of 51 volatiles were detected in 'Fuji' fruits. Ester compounds were a major part of total volatiles in the fruits. The highest total ester content was obtained from the trees on M9 rootstock, whereas the lowest was from MM106 rootstock. 2-Methylbutyl acetate and ethyl 2-methylbutanoate were the main ester compounds in the fruits considering all volatiles. The total ester concentration in the total volatile compounds in the 'Fuji' apples grafted on the M9 rootstock was $95.52 \%$. This rate was $91.28 \%$ for the M26 rootstock, 91.04\% for the MM111 rootstock and $88.30 \%$ for the MM106 rootstock. The study concluded that the M9 rootstock enhances the aroma content of 'Fuji' apples.
\end{abstract}

Keywords: pomology, quality, chromatography, ester compounds, SPME

\section{Introduction}

The total world apple production for 2016 was 89,329,179 metric tons. China, USA, Poland, Turkey, Iran and Italy had the greatest shares of apple production. In Turkey, apples are grown in almost all parts of the country, and there was a total 3,032,164 metric tons of apple production in 2017 (FAOSTAT, 2018). The most widely-grown varieties of apples in Turkey are 'Starking Delicious', 'Golden Delicious', 'Amasya', 'Jonathan', 'Granny Smith', 'Red Chief', 'Braeburn', 'Pink Lady', 'Gala' and 'Fuji' (Özçağıran, 2005). 'Fuji' apples are one of the popular apple varieties frequently grown mostly in China, Japan, Brazil, USA, New Zealand and Spain. Besides its high fruit quality, 'Fuji' apples are long-lasting fruits. It is reported that the significant aroma compounds in 'Fuji' fruits are ethyl 2-methylbutanoate, 2-methylbutyl acetate and hexyl acetate (Echeverría et al., 2004). Aroma has been known since ancient times (Vicentini et al., 2018). Aroma is of capital importance for quality characteristics in apples. Aroma is the foremost characteristic that influences the purchase decision of a consumer about apples. There are numerous studies on volatile compounds that contribute to the flavour of an apple and more than 300 volatile compounds have been identified through chromatography techniques (Dimick and Hoskin, 1983). One of these techniques, GC-MS, helps to investigate phenolic and polyphenolic compounds in plant species (Zishan et al., 2017). Ester compounds are the main components of apple flavour (Paillard, 1990).

The selection and use of rootstock is vital. It has great impact on the characteristics of the apple variety grafted on the rootstock. It also affects yield, biotic and abiotic 
stress conditions, and quality characteristics of fruits. Many researchers have examined and published several studies on the impact of rootstocks on yield and fruit quality in apples. The most widely used rootstocks in the world are M9, MM106, MM111, and M26 (Jackson, 2003). The M9 rootstock is the most commonly used dwarf rootstock for high density planting. Apple seedling rootstocks produce trees about 35 to 40 percent larger.

According to the literature, there is no specific research concerning the comparison of aroma compounds for different widely-used apple rootstocks. This study was performed in 2015-2016 and seeks to compare the aroma volatile compounds that contribute to the flavour of 'Fuji' variety grafted on different rootstocks.

\section{Materials and Methods}

The 'Fuji' orchard was established with a row and intra-row spacing of 3 meters to 1.5 meters for the M9 rootstock, 3 meters to 2 meters for the M26 rootstock, 4 meters to 3 meters for the MM106 rootstock and 6 meters to 5 meters for the MM111 rootstock in 2010. The orchards where the fruit samples were collected, are located in the district of Lapseki, Çanakkale, Turkey (Fig. 1).

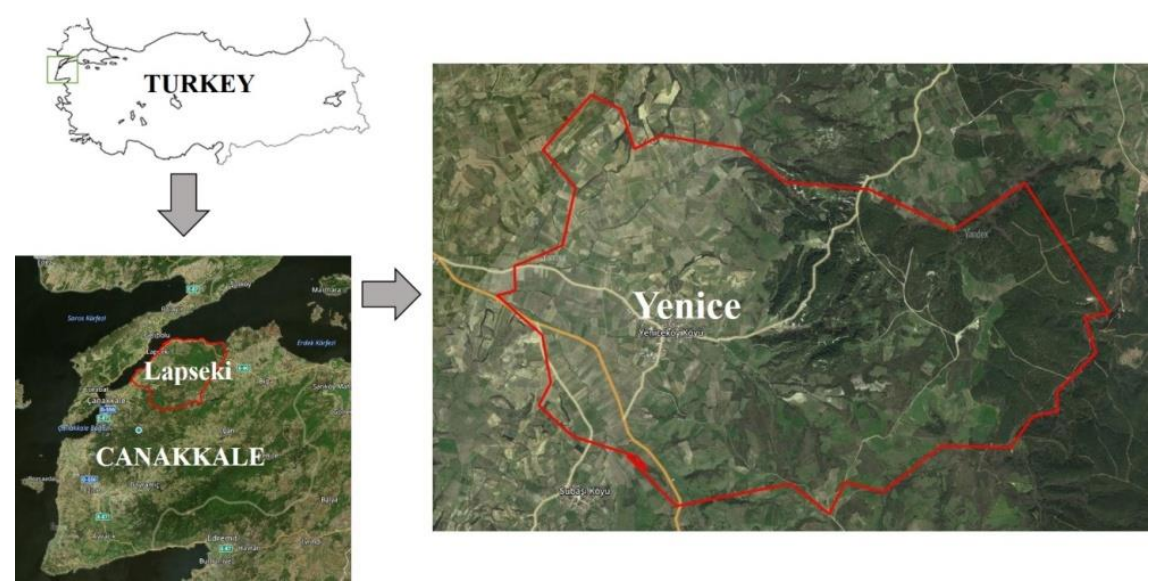

Figure 1. Study area

The region is well known for high quality pome fruits and stone fruits production and located at the nortwest of Turkey. In this region annual apple production is approxmately 115.000 tons. Different planting distances are used for commercial apple production due to rootstock effects on tree sizes. Application of fertilizers, pesticides, irrigation schedule were arranged according to the Global GAP certification programme for apple producers in Lapseki-Çanakkale. All the applications were made at the same time and same concentrations. The soil texture was generally clay-loamy and the reaction was slightly alkaline $(\mathrm{pH}$ 7.2). GPS coordinates of experimental site is $40^{\circ} 19^{\prime} 48^{\prime}$ ' $\mathrm{N}, 26^{\circ} 43^{\prime} 47^{\prime}$ ' $\mathrm{E}$ and elevation is $49 \mathrm{~m}$ above sea level.

\section{Collection of fruit samples}

The fruits of the 'Fuji' trees grafted on different rootstocks were collected in 30 September, in accordance with commercial harvest criteria. The fruits were sampled during 2015 and 2016 harvesting seasons. The fruits collected did not have any harmful 
symptoms or suffer from any disease. These fruits were then transferred to the laboratories and prepared for pomological and chromatography analyses. Five trees from each rootstock / Fuji combinations were used for fruit sampling and 20 fruits from each trees were harvested for further analyses. So, one hundred fruits were used for each rootstock / Fuji combinations.

\section{Evaluation of fruit quality characteristics}

Fruit weight of 'Fuji' apples (g) was calculated considering the average value of 20 fruits by individual weighing with a $0.01 \mathrm{~g}$ precision digital balance (Model 612, Sartorius). Flesh firmness was measured on two paired sides of each fruit by removing a $1 \mathrm{~mm}$ thick disk of skin from each side of the fruit using a penetrometer (Model FT327, Effegi). Total soluble solids (TSS) in the fruit juice were measured with a digital refractometer (PR-101, Atago) and expressed as ${ }^{\circ}$ Brix. Titratable acidity (TA) was determined by using a sample of the juice from 20 fruits. The juice samples were diluted with distilled water $(1: 2)$ and titrated to $\mathrm{pH} 8.2$ with a benchtop $\mathrm{pH}$ meter (InoLab pH 7110, WTW) with $0.1 \mathrm{~N} \mathrm{NaOH}$. The results were expressed as g malic $\mathrm{acid} / 100 \mathrm{~g}$ fresh weight (FW).

\section{Volatile extraction and GC/MS conditions}

For headspace sampling, SPME fibres coated with polydimethylsiloxanedivinylbenzene (Supelco Co., Bellefonte, PA, USA) were used as described before by Wang et al. (2009). The fibre was activated according to the manufacturer's instructions. The headspace solid phase microextraction method was used for the isolation and concentration of volatiles. For each extraction, $2 \mathrm{~g}$ of the pulp and $0.6 \mathrm{~g}$ $\mathrm{NaCl}$ were placed in a $4 \mathrm{ml}$ capped vial. The vial was placed in a $45^{\circ} \mathrm{C}$ water bath with a magnetic stirrer to warm to constant temperature, and the SPME fibre was exposed to the headspace of the sample to adsorb the analyses for $30 \mathrm{~min}$. The fibre was then introduced into the heated chromatograph injector port.

The identification and quantification of volatile compounds in the samples were performed on a Shimadzu QP2010 Plus GC-MS (Fig. 2) using the method described by Wang et al. (2009) with a slight modification.

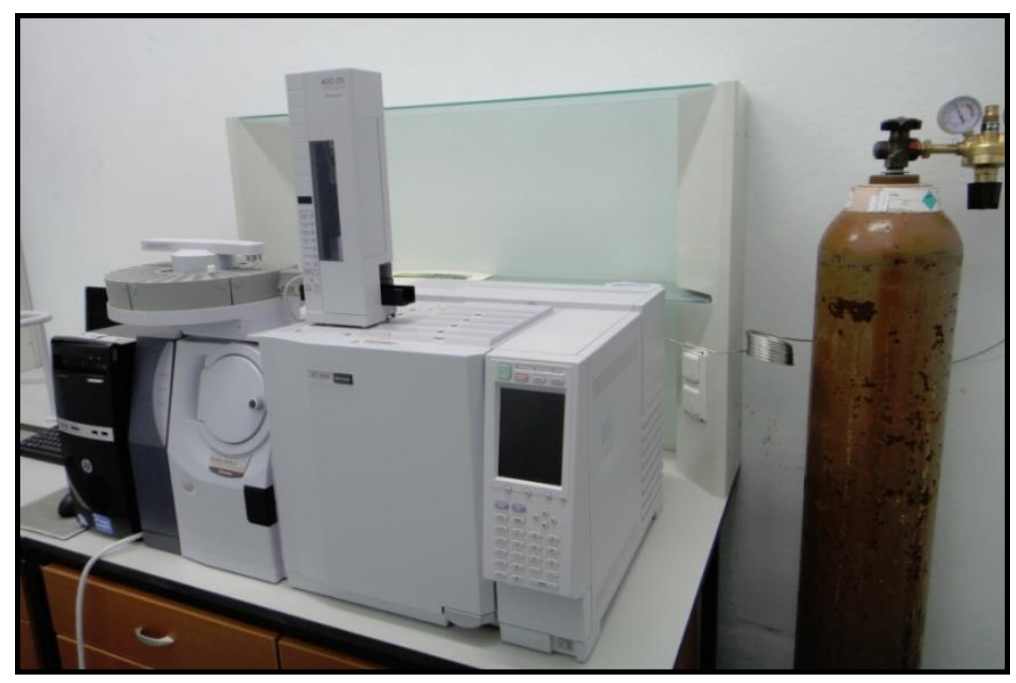

Figure 2. Shimadzu QP 2010 Plus GS-MS system for chromatography analyses of volatiles 
Separations were done with a DB-WAX column $(60 \mathrm{~m} \times 0.2 \mathrm{~mm}$, i.d., and $0.25 \mu \mathrm{m}$, film thickness; J \& W, USA), which was preconditioned at $250^{\circ} \mathrm{C}$ for a period of 2 hours. Helium was used as the carrier gas $(3 \mathrm{ml} / \mathrm{min})$. The analysis was conducted following the program at $40^{\circ} \mathrm{C}$ for $2 \mathrm{~min}, 40-150^{\circ} \mathrm{C}\left(3^{\circ} \mathrm{C} / \mathrm{min}\right)$, then $150-220^{\circ} \mathrm{C}$ $\left(10^{\circ} \mathrm{C} / \mathrm{min}\right)$, and $250^{\circ} \mathrm{C}(5 \mathrm{~min})$. The temperature of the injector was $250^{\circ} \mathrm{C}$. The interface between $\mathrm{GC}$ and MS was at $250^{\circ} \mathrm{C}$. Electron impact ionization was at $70 \mathrm{eV}$, EI mode, and the filament current was $0.25 \mathrm{~mA}$. The ion-source temperature was $200^{\circ} \mathrm{C}$. The scan range was $35-425$ aMU.

\section{Statistical analysis}

Differences between strains were evaluated with analysis of variance using the SAS General Linear Model procedure: PROC GLM procedure of SAS (version 6.12; SAS Institute, Cary NC) (SAS, 1997). When this analysis was statistically significant (Ftest), mean separation was performed using the Tukey Test.

\section{Results}

The 'Fuji' apple trees budded in mid-March under the ecological conditions where the study was performed, and came into full bloom by April $20^{\text {th }}$. The harvest was gathered on September $30^{\text {th }}$ in both years. The total number of days between full bloom and the harvest date was 152 to 158 days. Table 1 and Fig. 3 present the results from the pomological analysis of the fruit samples obtained in both years.

Table 1. Quality characteristics of the 'Fuji' apple fruits grafted on different rootstocks (average values of two years)

\begin{tabular}{c|c|c|c|c|c|c|c}
\hline & $\begin{array}{c}\text { Fruit weight } \\
(\mathbf{g})\end{array}$ & $\begin{array}{c}\text { Fruit width } \\
(\mathbf{m m})\end{array}$ & $\begin{array}{c}\text { Fruit length } \\
(\mathbf{m m})\end{array}$ & $\begin{array}{c}\text { Fruit } \\
\text { firmness }\end{array}$ & $\begin{array}{c}\text { Total soluble } \\
\text { solids (\%) }\end{array}$ & pH & $\begin{array}{c}\text { Total } \\
\text { titratable } \\
\text { acidity }(\%)\end{array}$ \\
\hline M9 & $212.20 \pm 4.26$ & $82.43 \pm 9.15$ & $67.59 \pm 8.45$ & $4.96 \pm 0.62$ & $18.40 \pm 1.63$ & $4.20 \pm 0.05$ & $0.48 \pm 0.02$ \\
M26 & $188.60 \pm 8.54$ & $78.30 \pm 5.55$ & $54.65 \pm 6.35$ & $6.75 \pm 0.65$ & $14.70 \pm 1.14$ & $4.10 \pm 0.03$ & $0.63 \pm 0.02$ \\
MM106 & $157.33 \pm 9.35$ & $71.74 \pm 7.25$ & $49.59 \pm 7.65$ & $7.00 \pm 0.54$ & $12.50 \pm 1.21$ & $4.70 \pm 0.08$ & $0.88 \pm 0.03$ \\
MM111 & $145.82 \pm 11.75$ & $68.43 \pm 8.35$ & $47.19 \pm 8.75$ & $4.96 \pm 0.57$ & $12.40 \pm 0.88$ & $3.80 \pm 0.09$ & $0.90 \pm 0.05$ \\
\hline LSD\%5 & 8.1 & 4.4 & 4.7 & 1.2 & 1.7 & 0.2 & 0.06 \\
\hline
\end{tabular}

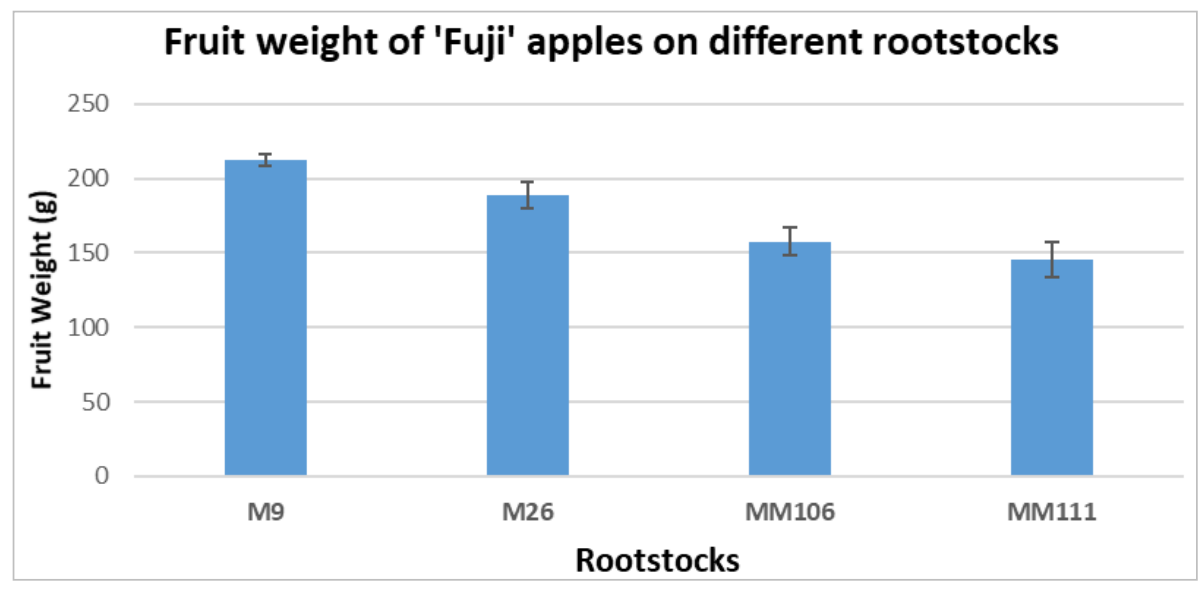

Figure 3. Comparison of fruit weight of 'Fuji' apples grafted on different rootstocks 
Among the 'Fuji' apple varieties grafted on different rootstocks, the fruits grafted on M9 were 212.20 grams in weight, which was the highest fruit weight. The apples with the highest fruit width were $82.43 \mathrm{~mm}$ grafted on M9, whereas those with the lowest fruit width were the ones grafted on MM111. Further, the apples with the highest fruit length were $67.59 \mathrm{~mm}$ grafted on M9, while those with lowest fruit length were $47.19 \mathrm{~mm}$ grafted on MM111. The fruits grafted on MM106 had the highest fruit firmness of 7.00; on the other hand, the fruits grafted on M9 and MM111 had lowest fruit firmness of 4.96. The fruits with the highest amount of soluble solids were grafted on the M9 rootstock with $18.40 \%$, while the fruits with the lowest amount of soluble solids were grafted on MM111 with $12.40 \%$. The highest $\mathrm{pH}$ value in the fruits was 4.70 grafted on MM106, while the lowest $\mathrm{pH}$ value in the fruits was 3.80 grafted on the MM111. The apples with the highest total acidity values were grafted on MM111 of $0.90 \%$, while the apples with the lowest total acidity values were grafted on M9 of $0.48 \%$.

The 'Fuji' apples grafted on M9 have an average fruit weight of 283.96 grams, fruit width of $84 \mathrm{~mm}$, fruit length of $73.6 \mathrm{~mm}, 12.96 \%$ soluble solids, $0.59 \%$ total acidity and $\mathrm{pH}$ of 3.91 (Baytekin and Akca, 2011). Another study found that the 'Fuji' variety grafted on the M9 rootstock had a fruit weight of 197.33 grams and $16 \%$ soluble solids (Ünivar and Pirlak, 2016). Various researchers have reported the positive effects of the M9 rootstock on fruit weight (Nicolai, 1998; Brown and Wolfe, 1999; Autio and Krupa, 2001; Marini, 2002).

\section{Volatile aroma compounds}

The chromatography analyses of the fruit samples obtained from the apple trees grafted on different rootstocks successfully revealed the volatile compounds. Table 2 presents the results for the volatile compounds and amounts of the samples from 2015. Table 3 shows the results of the samples from 2016; Table 4 presents the average results for both years.

Based on the results from 2015 and 2016, there were a total of 51 volatile compounds identified in both years. The aroma profiles in 2015 and 2016 were similar since the climate conditions in these two years were similar. The analyses concluded that 22 of the compounds were ester compounds; 17 of them were alcohol compounds; 7 of them were acid compounds and 5 were aldehyde compounds.

The total aroma amount of the fruits examined in the year 2015 varied between $275.13 \mu \mathrm{g} / \mathrm{kg}$ and $559.58 \mu \mathrm{g} / \mathrm{kg}$. The aroma compounds in the 'Fuji' apples on the M9 rootstock were 2.03 times more than those on the MM106 rootstock (Table 2). The total ester content in the 'Fuji' fruits on the M9 rootstock was $535.02 \mu \mathrm{g} / \mathrm{kg}$, whereas the MM106 rootstock had the lowest ester content of $242.29 \mu \mathrm{g} / \mathrm{kg}$. This was also the case for 2016 (Table 3). In 2016, the total volatile compounds from M9 amounted to $542.05 \mu \mathrm{g} / \mathrm{kg}$, while the total volatile compounds from MM106 amounted to $300.92 \mu \mathrm{g} / \mathrm{kg}$. As for the ester compounds, those from M9 were $517.34 \mu \mathrm{g} / \mathrm{kg}$ and those from MM106 were $266.49 \mu \mathrm{g} / \mathrm{kg}$. Considering the average values, the total volatile compounds in the 'Fuji' fruits on the M9 rootstock amounted to $550.89 \mu \mathrm{g} / \mathrm{kg}$. The M9 rootstock was followed by MM111 (409.16 $\mu \mathrm{g} / \mathrm{kg})$, M26 $(352.00 \mu \mathrm{g} / \mathrm{kg})$, and MM106 $(288.15 \mu \mathrm{g} / \mathrm{kg})$ (Table 4). That being said, it can be argued that compared to other rootstocks, the M9 rootstock has an enhancing effect on the aroma content of the 'Fuji' fruits (Fig. 4). 
Table 2. Volatile compounds of the 'Fuji' apple variety grafted on different rootstocks $(\mu \mathrm{g} / \mathrm{kg} \mathrm{FW})(2015)$

\begin{tabular}{|c|c|c|c|c|c|}
\hline \multirow{2}{*}{\multicolumn{2}{|c|}{ Esters }} & \multicolumn{4}{|c|}{ Rootstocks } \\
\hline & & \multirow{2}{*}{$\begin{array}{l}\text { M9 } \\
4.50\end{array}$} & \multirow{2}{*}{$\begin{array}{l}\text { M26 } \\
13.11\end{array}$} & \multirow{2}{*}{$\begin{array}{l}\text { MM106 } \\
14.46\end{array}$} & \multirow{2}{*}{$\begin{array}{l}\text { MM111 } \\
15.36\end{array}$} \\
\hline 1 & Ethyl acetate & & & & \\
\hline 2 & Propyl acetate & 2.43 & 2.14 & 4.23 & 1.66 \\
\hline 3 & 2-Methylpropyl acetate & 0.02 & 0.07 & 0.07 & 0.08 \\
\hline 4 & Butyl acetate & 1.29 & 1.70 & 1.32 & 1.99 \\
\hline 5 & 2-Methylbutyl acetate & 243.34 & 125.54 & 96.56 & 132.54 \\
\hline 6 & Pentyl acetate & 2.66 & 1.18 & 1.54 & 2.43 \\
\hline 7 & Butyl butanoate & 2.34 & 1.76 & 0.97 & 2.67 \\
\hline 8 & Hexyl acetate & 27.54 & 11.30 & 6.45 & 20.14 \\
\hline 9 & Hexyl butanoate & 4.56 & 3.11 & 2.76 & 3.50 \\
\hline 10 & Hexyl-2-methylbutanoate & 5.34 & 3.64 & 1.75 & 7.80 \\
\hline 11 & Hexyl hexanoate & 0.03 & 0.03 & 0.02 & 0.04 \\
\hline 12 & Ethyl formate & 0.28 & 0.86 & 1.12 & 1.07 \\
\hline 13 & Ethyl propanoate & 0.02 & 0.07 & 0.01 & 0.05 \\
\hline 14 & Tert-Butyl propanoate & 0.02 & 0.03 & 0.02 & 0.04 \\
\hline 15 & Butyl propanoate & 22.12 & 12.54 & 3.54 & 18.80 \\
\hline 16 & Hexyl propanoate & 13.34 & 8.90 & 5.76 & 20.45 \\
\hline 17 & Ethyl butanoate & 0.01 & 0.01 & 0.02 & 0.04 \\
\hline 18 & Hexyl butanoate & 3.36 & 2.16 & 3.34 & 4.43 \\
\hline 19 & Ethyl 2-methylbutanoate & 185.45 & 125.76 & 90.45 & 124.78 \\
\hline 20 & Butyl 2-methylbutanoate & 7.75 & 5.14 & 3.75 & 8.80 \\
\hline 21 & Ethyl hexanoate & 2.24 & 1.16 & 0.45 & 3.67 \\
\hline \multirow[t]{2}{*}{22} & Butyl hexanoate & 6.38 & 5.60 & 3.70 & 7.30 \\
\hline & TOTAL ESTERS & 535.02 & 325.81 & 242.29 & 377.64 \\
\hline \multicolumn{6}{|c|}{ Alcohols } \\
\hline 23 & 2-Propanol & 1.03 & 1.16 & 0.64 & 0.19 \\
\hline 24 & Ethanol & 4.17 & 3.54 & 4.30 & 5.63 \\
\hline 25 & 2-Butanol & 0.03 & 0.09 & 0.08 & 0.11 \\
\hline 26 & 2-Methyl-1-propanol & 0.16 & 0.35 & 0.35 & 0.41 \\
\hline 27 & 3-Pentanol & 0.02 & 0.09 & 0.13 & 0.11 \\
\hline 28 & 2-Pentanol & 0.07 & 0.27 & 0.4 & 0.32 \\
\hline 29 & Butanol & 6.73 & 8.10 & 6.62 & 9.47 \\
\hline 30 & 2-Methyl-1-butanol & 1.23 & 3.67 & 2.47 & 4.30 \\
\hline 31 & Pentanol & 0.20 & 0.26 & 0.25 & 0.30 \\
\hline 32 & Hexanol & 2.39 & 2.93 & 2.83 & 3.42 \\
\hline 33 & [Z]-3-hexen-1-ol & 0.02 & 0.03 & 0.04 & 0.04 \\
\hline 34 & {$[\mathrm{E}]-2$-hexen-1-ol } & 0.09 & 0.09 & 0.20 & 0.11 \\
\hline 35 & 2-Methyl-1-phenyl-1-propanol & 0.06 & 0.18 & 0.26 & 0.21 \\
\hline 36 & Farnesol & 0.12 & 0.11 & 0.19 & 0.13 \\
\hline 37 & Tetradecanol & 0.04 & 0.09 & 0.06 & 0.11 \\
\hline 38 & 6-Methyl-5-hepten-2-ol & 0.07 & 0.05 & 0.14 & 0.06 \\
\hline \multirow[t]{2}{*}{39} & 2-Ethyl-1-hexanol & 0.10 & 0.32 & 0.37 & 0.37 \\
\hline & TOTAL ALCOHOLS & 16.53 & 21.33 & 19.33 & 25.29 \\
\hline \multicolumn{6}{|c|}{ Acids } \\
\hline 40 & Acetic acid & 0.31 & 1.20 & 1.39 & 1.40 \\
\hline 41 & Hexanoic acid & 0.28 & 0.42 & 0.67 & 0.49 \\
\hline 42 & [E]-2-Hexenoic acid & 0.03 & 0.08 & 0.05 & 0.09 \\
\hline 43 & Octanoic acid & 0.02 & 0.07 & 0.08 & 0.08 \\
\hline 44 & Nonanoic acid & 0.02 & 0.07 & 0.04 & 0.08 \\
\hline 45 & Dodecanoic acid & 0.01 & 0.01 & 0.01 & 0.01 \\
\hline \multirow[t]{2}{*}{46} & Tridecanoic acid & 0.07 & 0.10 & 0.02 & 0.12 \\
\hline & TOTAL ACIDS & 0.74 & 1.95 & 2.26 & 2.27 \\
\hline \multicolumn{6}{|c|}{ Aldehydes } \\
\hline 47 & Hexanal & 4.07 & 3.88 & 6.03 & 4.53 \\
\hline 48 & [E]-2-hexenal & 3.01 & 3.37 & 4.84 & 3.94 \\
\hline 49 & 2-Methyl-4-pentenal & 0.06 & 0.07 & 0.11 & 0.08 \\
\hline 50 & Butanal & 0.07 & 0.19 & 0.16 & 0.22 \\
\hline \multirow[t]{3}{*}{51} & [Z]-3-hexenal & 0.08 & 0.09 & 0.11 & 0.11 \\
\hline & TOTAL ALDEHYDES & 7.29 & 7.60 & 11.25 & 8.88 \\
\hline & TOTAL VOLATILES & 559.58 & 356.69 & 275.13 & 414.08 \\
\hline
\end{tabular}


Table 3. Volatile compounds of the 'Fuji' apple variety grafted on different rootstocks $(\mu \mathrm{g} / \mathrm{kg} \mathrm{FW)} \mathrm{(2016)}$

\begin{tabular}{|c|c|c|c|c|c|}
\hline \multirow{2}{*}{\multicolumn{2}{|c|}{ Esters }} & \multicolumn{4}{|c|}{$\begin{array}{l}\text { Rootstocks } \\
\end{array}$} \\
\hline & & M9 & M26 & MM106 & MM111 \\
\hline 1 & Ethyl acetate & 4.19 & 11.93 & 14.03 & 14.44 \\
\hline 2 & Propyl acetate & 2.28 & 1.95 & 4.10 & 1.56 \\
\hline 3 & 2-Methylpropyl acetate & 0.02 & 0.06 & 0.07 & 0.08 \\
\hline 4 & Butyl acetate & 1.33 & 1.72 & 1.41 & 1.87 \\
\hline 5 & 2-Methylbutyl acetate & 250.64 & 126.8 & 113.32 & 124.59 \\
\hline 6 & Pentyl acetate & 2.74 & 1.19 & 1.65 & 2.50 \\
\hline 7 & Butyl butanoate & 2.41 & 1.78 & 1.04 & 2.75 \\
\hline 8 & Hexyl acetate & 28.37 & 11.41 & 6.50 & 20.74 \\
\hline 9 & Hexyl butanoate & 4.70 & 3.14 & 2.85 & 3.61 \\
\hline 10 & Hexyl-2-methylbutanoate & 5.50 & 3.68 & 1.87 & 8.03 \\
\hline 11 & Hexyl hexanoate & 0.03 & 0.03 & 0.02 & 0.04 \\
\hline 12 & Ethyl formate & 0.29 & 0.87 & 1.20 & 1.10 \\
\hline 13 & Ethyl propanoate & 0.02 & 0.07 & 0.01 & 0.05 \\
\hline 14 & Tert-Butyl propanoate & 0.02 & 0.03 & 0.02 & 0.04 \\
\hline 15 & Butyl propanoate & 20.57 & 12.67 & 3.79 & 19.36 \\
\hline 16 & Hexyl propanoate & 13.41 & 8.10 & 6.16 & 21.06 \\
\hline 17 & Ethyl butanoate & 0.01 & 0.01 & 0.02 & 0.04 \\
\hline 18 & Hexyl butanoate & 3.12 & 1.97 & 3.57 & 4.56 \\
\hline 19 & Ethyl 2-methylbutanoate & 162.47 & 118.44 & 96.78 & 121.52 \\
\hline 20 & Butyl 2-methylbutanoate & 7.21 & 4.68 & 4.01 & 9.06 \\
\hline 21 & Ethyl hexanoate & 2.08 & 1.06 & 0.48 & 3.45 \\
\hline \multirow[t]{2}{*}{22} & Butyl hexanoate & 5.93 & 5.10 & 3.59 & 6.86 \\
\hline & TOTAL ESTERS & 517.34 & 316.69 & 266.49 & 367.31 \\
\hline \multicolumn{6}{|c|}{ Alcohols } \\
\hline 23 & 2-Propanol & 0.96 & 1.06 & 0.62 & 0.18 \\
\hline 24 & Ethanol & 3.92 & 3.22 & 4.17 & 5.29 \\
\hline 25 & 2-Butanol & 0.03 & 0.08 & 0.08 & 0.10 \\
\hline 26 & 2-Methyl-1-propanol & 0.16 & 0.35 & 0.37 & 0.39 \\
\hline 27 & 3-Pentanol & 0.02 & 0.09 & 0.14 & 0.10 \\
\hline 28 & 2-Pentanol & 0.07 & 0.27 & 0.43 & 0.33 \\
\hline 29 & Butanol & 6.93 & 8.18 & 7.08 & 9.75 \\
\hline 30 & 2-Methyl-1-butanol & 1.27 & 3.71 & 2.64 & 4.43 \\
\hline 31 & Pentanol & 0.21 & 0.26 & 0.27 & 0.31 \\
\hline 32 & Hexanol & 2.46 & 2.96 & 3.03 & 3.52 \\
\hline 33 & [Z]-3-hexen-1-ol & 0.02 & 0.03 & 0.04 & 0.04 \\
\hline 34 & {$[\mathrm{E}]-2$-hexen-1-ol } & 0.09 & 0.09 & 0.21 & 0.11 \\
\hline 35 & 2-Methyl-1-phenyl-1-propanol & 0.06 & 0.18 & 0.28 & 0.22 \\
\hline 36 & Farnesol & 0.11 & 0.11 & 0.20 & 0.13 \\
\hline 37 & Tetradecanol & 0.04 & 0.09 & 0.06 & 0.11 \\
\hline 38 & 6-Methyl-5-hepten-2-ol & 0.07 & 0.05 & 0.15 & 0.06 \\
\hline \multirow[t]{2}{*}{$\begin{array}{l}50 \\
39 \\
\end{array}$} & 2-Ethyl-1-hexanol & 0.09 & 0.29 & 0.40 & 0.38 \\
\hline & TOTAL ALCOHOLS & 16.51 & 21.02 & 20.17 & 25.45 \\
\hline \multicolumn{6}{|c|}{ Acids } \\
\hline 40 & Acetic acid & 0.29 & 1.09 & 1.35 & 1.32 \\
\hline 41 & Hexanoic acid & 0.26 & 0.38 & 0.65 & 0.46 \\
\hline 42 & [E]-2-Hexenoic acid & 0.03 & 0.07 & 0.05 & 0.08 \\
\hline 43 & Octanoic acid & 0.02 & 0.07 & 0.09 & 0.08 \\
\hline 44 & Nonanoic acid & 0.02 & 0.07 & 0.04 & 0.08 \\
\hline 45 & Dodecanoic acid & 0.01 & 0.01 & 0.02 & 0.01 \\
\hline \multirow[t]{2}{*}{46} & Tridecanoic acid & 0.07 & 0.10 & 0.02 & 0.12 \\
\hline & TOTAL ACIDS & 0.7 & 1.79 & 2.22 & 2.15 \\
\hline \multicolumn{6}{|c|}{ Aldehydes } \\
\hline 47 & Hexanal & 4.19 & 3.92 & 6.45 & 4.67 \\
\hline 48 & [E]-2-hexenal & 3.10 & 3.4 & 5.18 & 4.06 \\
\hline 49 & 2-Methyl-4-pentenal & 0.06 & 0.07 & 0.12 & 0.08 \\
\hline 50 & Butanal & 0.07 & 0.19 & 0.17 & 0.23 \\
\hline \multirow[t]{3}{*}{51} & [Z]-3-hexenal & 0.08 & 0.09 & 0.12 & 0.11 \\
\hline & TOTAL ALDEHYDES & 7.5 & 7.67 & 12.04 & 9.15 \\
\hline & TOTAL VOLATILES & 542.05 & 347.17 & 300.92 & 404.06 \\
\hline
\end{tabular}


Table 4. Volatile compounds of the 'Fuji' apple variety grafted on different rootstocks $(\mu \mathrm{g} / \mathrm{kg} \mathrm{FW})$ (Average)

\begin{tabular}{|c|c|c|c|c|c|c|}
\hline \multirow{2}{*}{\multicolumn{2}{|c|}{ Esters }} & \multicolumn{5}{|c|}{ Rootstocks } \\
\hline & & \multirow{2}{*}{$\begin{array}{l}\text { M9 } \\
4.35 \mathrm{c}^{*}\end{array}$} & \multirow{2}{*}{$\begin{array}{l}\text { M26 } \\
12.52 \mathrm{~b}\end{array}$} & \multirow{2}{*}{$\begin{array}{l}\text { MM106 } \\
14.25 \mathrm{a}\end{array}$} & \multirow{2}{*}{$\begin{array}{l}\text { MM111 } \\
14.90 \mathrm{a}\end{array}$} & \multirow{2}{*}{$\begin{array}{l}\text { MSD* } \\
1.422\end{array}$} \\
\hline 1 & Ethyl acetate & & & & & \\
\hline 2 & Propyl acetate & $2.36 \mathrm{~b}$ & $2.05 \mathrm{c}$ & $4.17 \mathrm{a}$ & $1.61 \mathrm{~d}$ & 0.262 \\
\hline 3 & 2-Methylpropyl acetate & $0.02 \mathrm{c}$ & $0.07 \mathrm{~b}$ & $0.07 \mathrm{ab}$ & $0.08 \mathrm{a}$ & 0.010 \\
\hline 4 & Butyl acetate & $1.31 \mathrm{c}$ & $1.71 \mathrm{~b}$ & $1.37 \mathrm{c}$ & $1.93 \mathrm{a}$ & 0.140 \\
\hline 5 & 2-Methylbutyl acetate & $246.99 \mathrm{a}$ & $126.17 \mathrm{~b}$ & $104.94 \mathrm{c}$ & $128.57 \mathrm{~b}$ & 17.886 \\
\hline 6 & Pentyl acetate & $2.70 \mathrm{a}$ & $1.19 \mathrm{~d}$ & $1.60 \mathrm{c}$ & $2.47 \mathrm{~b}$ & 0.138 \\
\hline 7 & Butyl butanoate & $2.38 \mathrm{~b}$ & $1.77 \mathrm{c}$ & $1.01 \mathrm{~d}$ & $2.71 \mathrm{a}$ & 0.116 \\
\hline 8 & Hexyl acetate & $27.96 \mathrm{a}$ & $11.36 \mathrm{c}$ & $6.48 \mathrm{~d}$ & $20.44 \mathrm{~b}$ & 0.924 \\
\hline 9 & Hexyl butanoate & $4.63 \mathrm{a}$ & $3.13 \mathrm{c}$ & $2.81 \mathrm{~d}$ & $3.56 \mathrm{~b}$ & 0.181 \\
\hline 10 & Hexyl-2-methylbutanoate & $5.42 \mathrm{~b}$ & $3.66 \mathrm{c}$ & $1.81 \mathrm{~d}$ & $7.92 \mathrm{a}$ & 0.275 \\
\hline 11 & Hexyl hexanoate & 0.03 & 0.03 & 0.02 & 0.04 & NS \\
\hline 12 & Ethyl formate & $0.29 \mathrm{c}$ & $0.87 \mathrm{~b}$ & $1.16 \mathrm{a}$ & $1.09 \mathrm{a}$ & 0.078 \\
\hline 13 & Ethyl propanoate & 0.02 & 0.07 & 0.01 & 0.05 & NS \\
\hline 14 & Tert-Butyl propanoate & 0.02 & 0.03 & 0.02 & 0.04 & NS \\
\hline 15 & Butyl propanoate & $21.35 \mathrm{a}$ & $12.61 \mathrm{c}$ & $3.67 \mathrm{~d}$ & $19.08 \mathrm{~b}$ & 1.497 \\
\hline 16 & Hexyl propanoate & $13.38 \mathrm{~b}$ & $8.50 \mathrm{c}$ & $5.96 \mathrm{~d}$ & $20.76 \mathrm{a}$ & 0.972 \\
\hline 17 & Ethyl butanoate & 0.01 & 0.01 & 0.02 & 0.04 & NS \\
\hline 18 & Hexyl butanoate & $3.24 \mathrm{~b}$ & $2.07 \mathrm{c}$ & $3.46 \mathrm{~b}$ & $4.50 \mathrm{a}$ & 0.362 \\
\hline 19 & Ethyl 2-methylbutanoate & $173.96 \mathrm{a}$ & $122.10 \mathrm{~b}$ & $93.62 \mathrm{c}$ & $123.15 \mathrm{~b}$ & 22.518 \\
\hline 20 & Butyl 2-methylbutanoate & $7.48 \mathrm{~b}$ & $4.91 \mathrm{c}$ & $3.88 \mathrm{~d}$ & $8.93 \mathrm{a}$ & 0.716 \\
\hline 21 & Ethyl hexanoate & $2.16 \mathrm{~b}$ & $1.11 \mathrm{c}$ & $0.47 \mathrm{~d}$ & $3.56 \mathrm{a}$ & 0.261 \\
\hline \multirow[t]{2}{*}{22} & Butyl hexanoate & $6.16 \mathrm{~b}$ & $5.35 \mathrm{c}$ & $3.65 \mathrm{~d}$ & $7.08 \mathrm{a}$ & 0.727 \\
\hline & TOTAL ESTERS & 526.22 a & 321.29 c & $254.45 \mathrm{~d}$ & $372.51 \mathrm{~b}$ & 29.538 \\
\hline \multicolumn{7}{|c|}{ Alcohols } \\
\hline 23 & 2-Propanol & $1.00 \mathrm{~b}$ & $1.11 \mathrm{a}$ & $0.63 \mathrm{c}$ & $0.19 \mathrm{~d}$ & 0.111 \\
\hline 24 & Ethanol & $4.05 \mathrm{~b}$ & $3.38 \mathrm{c}$ & $4.24 \mathrm{~b}$ & $5.46 \mathrm{a}$ & 0.488 \\
\hline 25 & 2-Butanol & $0.03 \mathrm{~b}$ & $0.09 \mathrm{ab}$ & $0.08 \mathrm{ab}$ & $0.11 \mathrm{a}$ & 0.075 \\
\hline 26 & 2-Methyl-1-propanol & $0.16 \mathrm{~b}$ & $0.35 \mathrm{a}$ & $0.36 \mathrm{a}$ & $0.40 \mathrm{a}$ & 0.147 \\
\hline 27 & 3-Pentanol & $0.02 \mathrm{~b}$ & $0.09 \mathrm{a}$ & $0.14 \mathrm{a}$ & $0.11 \mathrm{a}$ & 0.057 \\
\hline 28 & 2-Pentanol & $0.07 \mathrm{~b}$ & $0.27 \mathrm{ab}$ & $0.42 \mathrm{a}$ & $0.33 \mathrm{a}$ & 0.214 \\
\hline 29 & Butanol & $6.83 \mathrm{c}$ & $8.14 \mathrm{~b}$ & $6.85 \mathrm{c}$ & $9.61 \mathrm{a}$ & 0.519 \\
\hline 30 & 2-Methyl-1-butanol & $1.25 \mathrm{~d}$ & $3.69 \mathrm{~b}$ & $2.56 \mathrm{c}$ & $4.37 \mathrm{a}$ & 0.198 \\
\hline 31 & Pentanol & 0.21 & 0.26 & 0.26 & 0.31 & NS \\
\hline 32 & Hexanol & $2.43 \mathrm{c}$ & $2.95 \mathrm{~b}$ & $2.93 \mathrm{~b}$ & $3.47 \mathrm{a}$ & 0.212 \\
\hline 33 & [Z]-3-hexen-1-ol & 0.02 & 0.03 & 0.04 & 0.04 & NS \\
\hline 34 & [E]-2-hexen-1-ol & $0.09 \mathrm{~b}$ & $0.09 \mathrm{~b}$ & $0.21 \mathrm{a}$ & $0.11 \mathrm{~b}$ & 0.079 \\
\hline 35 & 2-Methyl-1-phenyl-1-propanol & $0.06 \mathrm{c}$ & $0.18 \mathrm{~b}$ & $0.27 \mathrm{a}$ & $0.22 \mathrm{ab}$ & 0.081 \\
\hline 36 & Farnesol & $0.12 \mathrm{~b}$ & $0.11 \mathrm{~b}$ & $0.20 \mathrm{a}$ & $0.13 \mathrm{~b}$ & 0.059 \\
\hline 37 & Tetradecanol & 0.04 & 0.09 & 0.06 & 0.11 & NS \\
\hline 38 & 6-Methyl-5-hepten-2-ol & 0.07 & 0.05 & 0.15 & 0.06 & NS \\
\hline \multirow[t]{2}{*}{39} & 2-Ethyl-1-hexanol & $0.10 \mathrm{c}$ & $0.31 \mathrm{~b}$ & $0.39 \mathrm{a}$ & $0.38 \mathrm{a}$ & 0.041 \\
\hline & TOTAL ALCOHOLS & $16.55 \mathrm{~d}$ & $21.19 \mathrm{~b}$ & $19.79 \mathrm{c}$ & 25.41 a & 0.815 \\
\hline \multicolumn{7}{|c|}{ Acids } \\
\hline 40 & Acetic acid & $0.30 \mathrm{c}$ & $1.15 \mathrm{~b}$ & $1.37 \mathrm{a}$ & $1.36 \mathrm{a}$ & 0.128 \\
\hline 41 & Hexanoic acid & $0.27 \mathrm{~d}$ & $0.40 \mathrm{c}$ & $0.66 \mathrm{a}$ & $0.48 \mathrm{~b}$ & 0.052 \\
\hline 42 & [E]-2-Hexenoic acid & 0.03 & 0.08 & 0.05 & 0.09 & NS \\
\hline 43 & Octanoic acid & $0.02 \mathrm{~b}$ & $0.07 \mathrm{ab}$ & $0.09 \mathrm{a}$ & $0.08 \mathrm{a}$ & 0.055 \\
\hline 44 & Nonanoic acid & 0.02 & 0.07 & 0.04 & 0.08 & NS \\
\hline 45 & Dodecanoic acid & 0.01 & 0.01 & 0.02 & 0.01 & NS \\
\hline \multirow[t]{2}{*}{46} & Tridecanoic acid & $0.07 \mathrm{ab}$ & $0.10 \mathrm{ab}$ & $0.02 \mathrm{~b}$ & $0.12 \mathrm{a}$ & 0.081 \\
\hline & TOTAL ACIDS & $0.72 \mathrm{c}$ & $1.88 \mathrm{~b}$ & $2.25 \mathrm{a}$ & $2.22 \mathrm{a}$ & 0.186 \\
\hline \multicolumn{7}{|c|}{ Aldehydes } \\
\hline 47 & Hexanal & $4.13 \mathrm{c}$ & $3.90 \mathrm{c}$ & $6.24 \mathrm{a}$ & $4.60 \mathrm{~b}$ & 0.412 \\
\hline 48 & {$[\mathrm{E}]-2$-hexenal } & $3.06 \mathrm{c}$ & $3.39 \mathrm{c}$ & $5.01 \mathrm{a}$ & $4.00 \mathrm{~b}$ & 0.334 \\
\hline 49 & 2-Methyl-4-pentenal & $0.06 \mathrm{~b}$ & $0.07 \mathrm{~b}$ & $0.12 \mathrm{a}$ & $0.08 \mathrm{ab}$ & 0.045 \\
\hline 50 & Butanal & $0.07 \mathrm{~b}$ & $0.19 \mathrm{a}$ & $0.17 \mathrm{a}$ & $0.23 \mathrm{a}$ & 0.066 \\
\hline \multirow[t]{3}{*}{51} & [Z]-3-hexenal & 0.08 & 0.09 & 0.12 & 0.11 & NS \\
\hline & TOTAL ALDEHYDES & $7.40 \mathrm{c}$ & $7.64 \mathrm{c}$ & $11.66 \mathrm{a}$ & $9.02 \mathrm{~b}$ & 0.773 \\
\hline & TOTAL VOLATILES & 550.89 a & $352.00 \mathrm{c}$ & $288.15 d$ & $409.16 \mathrm{~b}$ & 30.544 \\
\hline
\end{tabular}

*MSD: Minimum Significant Difference on Tukey multiple range test by $p<0.01$, NS: Non-Significant 
The total ester concentration in the total volatile compounds of the 'Fuji' apples grafted on the M9 rootstock was $95.52 \%$. This rate was $91.28 \%$ for the M26 rootstock, $91.04 \%$ for the MM111 rootstock and $88.30 \%$ for the MM106 rootstock. Ester compounds are the main components of apple flavours and constitute 85 to $95 \%$ of the total volatile compounds (Paillard, 1990). Isoleucine, an amino acid, plays an important role in the synthesis of ester compounds. A greater availability of isoleucine amino acid increases the concentration of ester compounds (Sugimoto et al., 2011). Thus, it seems that the dwarf M9 rootstock has an increasing effect on the amount of isoleucine.

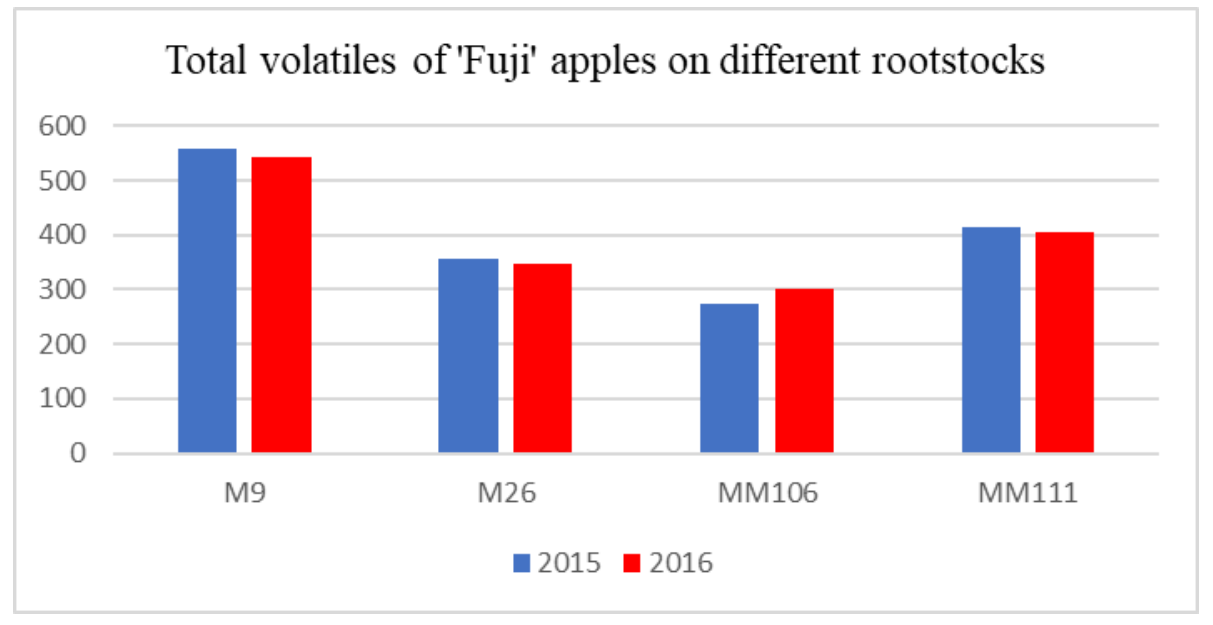

Figure 4. Total volatiles of 'Fuji' apples on different rootstocks in two years

In the year 2015, the ratio of 2-methylbutyl acetate compound in the 'Fuji' varieties grafted on M9 was $45.48 \%$ in all ester compounds and $43.49 \%$ in all volatile compounds; in 2016, this rate was $48.45 \%$ in all ester compounds and $46.24 \%$ in all volatile compounds. Further, in the year 2015, the ratio of 2-methylbutyl acetate compound in the 'Fuji' varieties grafted on M26 was $38.53 \%$ in all ester compounds and $35.20 \%$ in all volatile compounds; in 2016, this rate was $40.04 \%$ in all ester compounds and $36.52 \%$ in all volatile compounds. As for the MM106 rootstock, the ratio was $39.85 \%$ in all ester compounds and $35.10 \%$ in all volatile compounds in 2015 , and it was $45.52 \%$ in all ester compounds and $37.66 \%$ in all volatile compounds in 2016. Lastly, for the MM111 rootstock, the ratio was $35.10 \%$ in all ester compounds and $32.01 \%$ in all volatile compounds in 2015, and it was $33.92 \%$ in all ester compounds and $30.83 \%$ in all volatile compounds in 2016 . The other important ester compound in the 'Fuji' apples was ethyl 2-methylbutanoate. In the fruit samples obtained in 2015, the amount of this compound was $185.45 \mu \mathrm{g} / \mathrm{kg}$ for the M9 rootstock, $125.76 \mu \mathrm{g} / \mathrm{kg}$ for the M26 rootstock, $124.78 \mu \mathrm{g} / \mathrm{kg}$ for the MM111 rootstock, and $90.45 \mu \mathrm{g} / \mathrm{kg}$ for the MM106 rootstock (Table 2). In 2016, the amount was $162.47 \mu \mathrm{g} / \mathrm{kg}$ for the M9 rootstock, $121.52 \mu \mathrm{g} / \mathrm{kg}$ for the MM111 rootstock, $118.44 \mu \mathrm{g} / \mathrm{kg}$ for the M26 rootstock, and $96.78 \mu \mathrm{g} / \mathrm{kg}$ for the MM106 rootstock (Table 3). In the year 2015, the ratio of ethyl 2-methylbutanoate in the 'Fuji' varieties grafted on M9 was 34.66\% in all ester compounds and $33.14 \%$ in all volatile compounds; in 2016, this rate was $31.40 \%$ in all ester compounds and $29.97 \%$ in all volatile compounds. Further, in the year 2015 , the ratio of ethyl 2-methylbutanoate compound in the 'Fuji' varieties grafted on MM106, where the compound was found at the lowest amount, was $37.33 \%$ in all ester 
compounds and $32.88 \%$ in all volatile compounds; in 2016, this rate was $36.32 \%$ in all ester compounds and $32.16 \%$ in all volatile compounds. The average amount of the compound was $173.96 \mu \mathrm{g} / \mathrm{kg}$ for the M9 rootstock, $123.15 \mu \mathrm{g} / \mathrm{kg}$ for the MM111 rootstock, $122.10 \mu \mathrm{g} / \mathrm{kg}$ for the M26 rootstock and $93.62 \mu \mathrm{g} / \mathrm{kg}$ for the MM106 rootstock (Fig. 5).

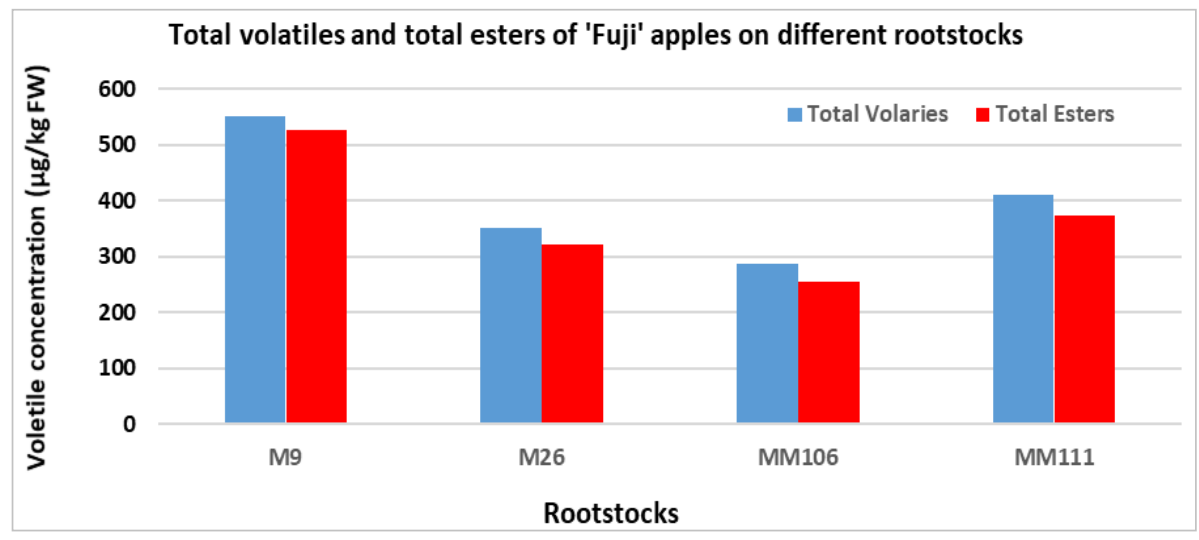

Figure 5. Comparison of total volatiles and total ester concentrations of 'Fuji' apples grafted on different rootstocks in two years

Besides, hexyl acetate and butyl propanoate are also notable among the ester compounds. Based on the average amount of these compounds, the 'Fuji' fruits obtained from the M9 rootstock yielded different results compared to other rootstocks (Table 4). Several researchers reported that esters are the main component groups of apple flavour (Echeverría et al., 2004; Mannucci et al., 2017). A study by Qin et al. (2017) identified 64 compounds based on the aroma profile of 'Fuji' apples gathered from 43 different regions in China. The researchers identified 43 ester compounds and the proportion of ester compounds in the total aroma compounds was $89.9 \%$. As for the total amount of alcohol, the fruits collected from the MM111 rootstock had the highest amount of alcohol in both years $(25.29$ and $25.45 \mu \mathrm{g} / \mathrm{kg})$. The M9 rootstock had the lowest amount of alcohol, which was $16.53 \mu \mathrm{g} / \mathrm{kg}$ and $16.51 \mu \mathrm{g} / \mathrm{kg}$, respectively (Table 2 and Table 3). Lower amounts of acidic compounds, which are among volatile compounds, were obtained compared to the amounts of the other main compound groups. In terms of the total acidic compounds, acetic acid identified in the MM111 rootstock was highest (Table 4). The amount of acetic acid in this rootstock was $1.40 \mu \mathrm{g} / \mathrm{kg}$ in 2015 and $1.32 \mu \mathrm{g} / \mathrm{kg}$ in 2016 (Table 2 and Table 3).

Following esters and alcohols, aldehydes were the volatile compound group with the next highest level. Hexanal and [E]-2-hexanal were the compounds with the highest values in this group. The MM106 rootstock had the highest average value in terms of the total amount of aldehyde compounds $(11.66 \mu \mathrm{g} / \mathrm{kg})$. The lowest amount of aldehyde was found in the fruits on the M9 rootstock $(7.40 \mu \mathrm{g} / \mathrm{kg})($ Table 4).

The results of this two-year study showed that the M9 rootstock has positive impact on the aroma profile of the 'Fuji' apples. It is remarkable that the M9 rootstock considerably increases the amount of ester compounds particularly. 


\section{Discussion}

Wang et al. (2005) analysed the volatile compounds of conventional and organically cultivated 'Fuji' apple varieties on GC-MS using SPME method. As a result, 64 compounds were found in organic system and 51 compounds were found in control fruits. Tao et al. (2011) used the GC-MS with SPME method to determine the aroma components in eight apple varieties. As a result, 28 esters, 3 alcohols and 2 alkane compounds were determined. A study by Kviklys et al. (2014) revealed that the total phenol content of the M9 rootstock was higher than the M26 rootstock. However, they reported that there was no distinctive difference between different rootstocks in terms of the total phenol content. Unlike the total phenolic compounds, the amount of volatile compounds varied between rootstocks.

Although the effect of rootstocks on the quality characteristics of fruits has been analysed in different varieties, the studies about their impact on aroma profile are extremely low in number. Seker et al. (2017) reported the positive effects of the 'GF677' rootstock on the aroma profile of 'Cresthaven', a peach variety. Further studies may focus on the effect of rootstocks on aroma profile in different apple varieties.

\section{Conclusion}

Rootstocks may have great effect on aroma compound formation due to different carbohydrate and fatty acid metabolism pathways. So, utilization of different rootstocks should be considered for specific aroma compound improvements in apples.

\section{REFERENCES}

[1] Autio, W. R., Krupa, J. (2001): Rootstock effects on ginger gold apple trees. - Fruit Notes 66: 50-52.

[2] Baytekin, S., Akca, Y. (2011): Determination of performance different apple cultivars on M9 apple rootstock. - Journal of Agricultural Faculty of Gaziosmanpasa University 28(1): 45-51.

[3] Brown, G. R., Wolfe, D. (1999): Rootstock and interstem effects on pome and stone fruit trees. - In: Rowell, B. (ed.) Fruit and Vegetable Crops Research Reports. Kentucky Agricultural Experiment Station, University of Kentucky College of Agriculture Department of Horticulture Lexington, p: 14-18.

[4] Dimick, P. S., Hoskin, J. C. (1983): Review of apple flavor-state of the art. - CRC Critical Reviews in Food Science and Nutrition 18: 387-409.

[5] Echeverría, G., Graell, J., López, M. L., Lara, I. (2004): Volatile production, quality and aroma-related enzyme activities during maturation of 'Fuji' apples. - Postharvest Biology and Technology 31(3): 217-227. doi:10.1016/j.postharvbio.2003.09.003.

[6] FAOSTAT. (2018): Food and Agricultural Organization of the United Nations, Statistical Data. - Available at : http://www.fao.org/faostat/en/\#data/PP.

[7] Jackson, J. E. (2003): Biology of apples and pears. - Cambridge University Press.

[8] Kvikly, D., Liaudanskas, M., Janulis, V., Viskelis, P., Rubinskiene, M., Lanauskas, J., Uselis, N. (2014): Rootstock genotype determines phenol content in apple fruits. - Plant, Soil and Environment 60(5): 234-240.

[9] Mannucci, A., Serra, A., Remorini, D., Castagna, A., Mele, M., Scartazza, A., Ranieri, A. (2017): Aroma profile of Fuji apples treated with gelatin edible coating during their storage. - LWT-Food Science and Techology 85: 28-36. 
[10] Marini, R. P. (2002): Does rootstock influence apple fruit size? - Compact Fruit Tree 35(1): 8-10.

[11] Nicolai, J. (1998): European trends in apple tree density, Rootstocks and Tree Training. 41 th Annual IDFTA Conference, February 21-25, Pasco, Washington.

[12] Özçağıran, R., Ünal, A., Özeker, E., İsfendiyaroğlu, M. (2005): Apple. Temperate Climate Fruit Types, Pome Fruits, Vol: II. - Ege University Faculty of Agriculture Publications, Bornova, İzmir, No: 556.

[13] Paillard, N. M. M. (1990): The flavour of apples, pears and quinces. - In: Morton, I. D., Macleod, A. J. (eds.) Food flavours, Part C. The flavour of fruits. Elsevier, Amsterdam, The Netherlands, p. 1-41.

[14] Qin, L., Wei, Q. P., Kang, W. H., Zhang, Q., Sun, J., Liu, S. Z. (2017): Comparison of volatile compounds in 'Fuji' apples in the different regions in China. - Food Science and Technology Research 23(1): 79-89.

[15] SAS Institute. (1997): SAS/STAT Guide for Personal Computers. - SAS Institute Inc., Cary, USA.

[16] Seker, M., Ekinci, N., Gur, E. (2017): Effects of different rootstocks on aroma volatile constituents in the fruits of peach (Prunus persica L. Batsch cv. 'Cresthaven'). - New Zealand Crop and Horticultural Science 45(1): 1-13.

[17] Sugimoto, N., Jones, A. D., Beaudry, R. (2011): Changes in free amino acid content in 'Jonagold' apple fruit as related to branched-chain ester production, ripening, and senescence. - J. Amer. Soc. Hort. Sci.

[18] Tao, C., Wang, D., Yang, X., Luo, Y. (2011): Determination of aroma components of 8 cultivars of apple fruit by SPME and GC-MS. - Journal of Gansu Agricultural University 2011-01.

[19] Ünüvar, G., Prrlak, L. (2016): Determination of phenological and pomological characters of some apple cultivars grafted on M9 rootstock at Karaman ecological conditions. Nevsehir Journal of Science And Technology, TARGID, Special Number: 96-106. DOI: 10.17100/nevbiltek.210971.

[20] Vicentini, C. B., Buldrini, F., Bosi, G., Romagnoli, C. (2018): "Spigo nardo": from the Erbario Estense a possible solution for its taxonomical attribution. - Rendiconti Lincei, Scienze Fisiche e Naturali 29(4): 909-921.

[21] Wang, X., Shi, D., Şark1, Y., Zhai, H. (2005): GC-MS analysis of fruit aroma components of organic 'Fuji' apple. - Journal of Fruit Science 2005-6.

[22] Wang, Y. J., Yang, C. X., Li, S. H., Yang, L., Wang, Y. N., Zhao, J. B., Jiang, Q. (2009): Volatile characteristics of 50 peaches and nectarines evaluated by HP-SPME with GCMS. - Food Chem. 116: 356-364.

[23] Zishan, A., Anwar, S., Shiwali, S. (2017): Evaluation of in vitro antioxidant activity, HPLC and GC-MS analysis along with chemoprofiling of Decalepis arayalpathra: a critically endangered plant of Western Ghats, India. - Rendiconti Lincei 28(4): 711-720. 